\title{
Sensitivity Analysis of Width Representation for Gait Recognition
}

\section{Sungjun Hong and Euntai Kim}

School of Electrical and Electronic Engineering, Yonsei University, Seoul, Korea

\section{]jfis}

\begin{abstract}
In this paper, we discuss a gait representation based on the width of silhouette in terms of discriminative power and robustness against the noise in silhouette image for gait recognition. Its sensitivity to the noise in silhouette image are rigorously analyzed using probabilistic noisy silhouette model. In addition, we develop a gait recognition system using width representation and identify subjects using the decision level fusion based on majority voting. Experiments on CASIA gait dataset A and the SOTON gait database demonstrate the recognition performance with respect to the noise level added to the silhouette image.
\end{abstract}

Keywords: Gait recognition, Biometrics, Human identification, Width vector, Sensitivity analysis

\section{Introduction}

Human identification systems utilizing biometrics have been implemented in many applications which require a high level of security, for example, visual surveillance, access control at airports, banks, and etc. [1]. There are various biometric features for human identification. Some examples include face, iris, fingerprint, and palmprint. Particularly, gait recognition system has attracted increasing interest since it has unique advantages over other biometrics [2]. For example, it is not intrusive and may be acquired at a distance. Also, it can be captured at low resolution. Gaits can thus be used in situations when other biometrics might not be applicable.

Gait analysis can mainly be classified under two major approaches: model-based approach and model-free approach [2]. The model-based approach adopts a human model and maps those extracted image features onto the components of the human model. Unfortunately, the model-based approaches require high quality gait sequences and high computational cost to recover human parameters and obtain good performance. Further, the approaches are limited by the imperfect computer vision techniques available for human modeling as well as the high computational cost incurred. Johnson and Bobick [3] proposed a multi-view gait recognition method using recovered static body parameters such as the height, the distance between head and pelvis, and the distance between the feet, which are measurements taken from static gait frames. Lee and Grimson [4] described a moment-based representation of gait appearance for the purpose of person identification. The silhouette of a walking person was divided into seven regions. Ellipses were fit to each region and region feature vector were formed, including averages of the centroid and the aspect ratio, etc. In [5], a more detailed model was proposed 
using ellipse for the torso and the head, line segments for the legs, and a rectangle for each foot.

In contrast, the model-free approaches act directly on a video sequence and characterize the walking pattern by signal analysis. Unlike the model-based approach, the model-free approach does not assume any specific human model, thereby saving the computational resources. Thus, model free approaches are considered further in this paper. Han and Bhanu [6] proposed a model-free gait representation, called gait energy image (GEI), and derived real and synthetic templates for gait. Fusion of synthetic and real templates was used to obtain the final recognition results. And Yang et al. [7] obtained the dynamic region in GEI which reflects the walking manner of an individual, called enhanced GEI. Li et al. [8] separate a silhouette into seven components, and perform human gait recognition first based on each of the seven components individually and on certain combinations of the seven components. Wang et al. [9] used the outer contour directly instead of the whole silhouette and unwrapped it into a distance signal to recognize a person. Kale et al. $[10,11]$ proposed a gait representation called the width vector. The width of the outer contour of a silhouette image is used as a gait feature in their works. Hong et al. [12] proposed multiple gait representation, called multi-bipolarized contour vector, inspired by Kale et al. [10] and combined them to identify walking person. Otherwise Liu et al. [13] used the horizontal and vertical projections of silhouettes as a gait representation and Hong et al. [14] presented a kind of the projection presentation, called the mass vector. Lam et al. [15] proposed gait flow image (GFI) generated by using an optical flow field without constructing any model.

In this paper, a theoretical analysis is conducted regarding the noise sensitivity of the width gait representation. Especially, the expectation of the width vector for silhouette noise is rigorously derived based on a probabilistic noise model and experimentally verified through simulation. Furthermore, we present a gait recognition system to divide a given gait sequence into complete gait cycles using the norm of the width vectors. The CASIA gait dataset A and the SOTON gait database are used to evaluate the recognition performance with respect to the noise level added to the silhouette image.

The rest of the paper is organized as follows. Section 2 describes the width vector, which is gait representation based on the width of the outer contour of a silhouette image. Section 3 presents a probabilistic noise model in a silhouette image and analyzes the sensitivity of the width vector with respect to silhouette noise. Section 4 presents a gait recognition system for human identification. Section 5 reports the experimental results on two gait databases. Finally, conclusions are drawn in Section 6.

\section{Gait Representation}

Individual walking pattern varies in terms of period, width, and distribution due to the diversity of the geometrical configurations of different individuals. Kale et al. [10, 11] used the width of the outer contour of a silhouette image as a gait feature in their work where the feature is called as the width vector. The width vector is defined as the difference between the $x$-coordinate values of the leftmost nonzero pixel and the rightmost nonzero pixel in row of a silhouette image. Thus, for a given $\mathrm{W} \times \mathrm{H}$ sized silhouette image, the width vector can be written mathematically as

$$
w(t)=\left[w_{1}(t), w_{2}(t), \ldots, w_{H}(t)\right] \in \mathbb{R}^{H},
$$

where $w_{h}(t)=x_{h}^{R}(t)-x_{h}^{L}(t) \geq 0$, for $\forall h=1,2, \ldots, H$ is the difference in location of the rightmost boundary pixel $x_{h}^{R}(t)$ and the leftmost boundary pixel $x_{h}^{L}(t)$ for a given row $h$ in the $t$ th frame.

Instead of using the width vector directly as in [10], the arithmetic mean of the width is employed as a feature vector to save the high cost of the frame-by-frame matching such as dynamic time wrapping (DTW). Explicitly, we calculate the mean of width vectors as

$$
\bar{w}=\frac{1}{T} \sum_{t=1}^{T} w(t) \in \mathbb{R}^{H},
$$

with $T$ being the number of frames in a gait sequence. The samples of the normalized silhouette images in a gait sequence for a single person and their corresponding width vectors are shown in Figure 1(a) and 1(b). The rightmost image in Figure 1 (b) is the mean of width vectors $\bar{w}$ for a given sequence. As shown in Figure 1, the width vector seems sensitive to structural differences and might be vulnerable to spurious pixels in a silhouette image.

\section{Sensitivity Analysis}

\subsection{Noise in Silhouette Image}

Let us denote an ideal noise-free silhouette image by $I_{x y}(t)$, an actual binary silhouette image and added noise by $N_{x y}(t)$ and $\eta_{x y}(t)$, respectively. Then, the actual silhouette $N_{x y}(t)$ is 


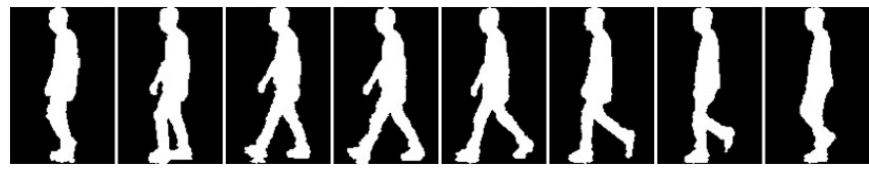

(a)

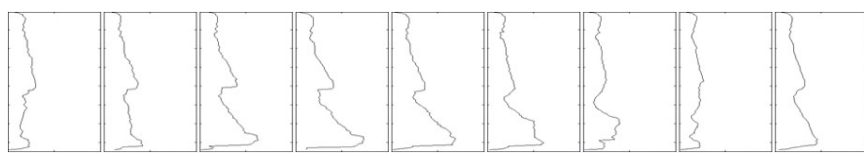

(b)

Figure 1. (a) Examples of normalized silhouette images in a gait sequence for a single person (b) the corresponding width vectors $w(t)$. The rightmost images in (b) is the mean of width vectors $\bar{w}$.

represented as

$$
N_{x y}(t)=I_{x y}(t)+\eta_{x y}(t) .
$$

For simplicity, it is assumed that the added noise $N_{x y}(t)$ be is identically and independently distributed (i.i.d.) and has the following distribution [16]:

$$
\eta_{x y}(t)=\left\{\begin{array}{rl}
\eta_{x y}^{1}(t): & \mathrm{P}\left(\eta_{x y}(t)=-1\right)=\varepsilon, \\
& \mathrm{P}\left(\eta_{x y}(t)=0\right)=1-\varepsilon, \text { if } I_{x y}(t)=1 \\
\eta_{x y}^{0}(t): & \mathrm{P}\left(\eta_{x y}(t)=1\right)=\varepsilon, \\
& \mathrm{P}\left(\eta_{x y}(t)=0\right)=1-\varepsilon, \text { if } I_{x y}(t)=0
\end{array} .\right.
$$

Then, we obtain

$$
E\left[\eta_{x y}(t)\right]=\left\{\begin{array}{ll}
-\varepsilon, & \text { if } I_{x y}(t)=1 \\
\varepsilon, & \text { if } I_{x y}(t)=0
\end{array},\right.
$$

and

$$
\operatorname{Var}\left[\eta_{x y}(t)\right]=\operatorname{Var}\left[\eta_{x y}^{1}(t)\right]=\operatorname{Var}\left[\eta_{x y}^{0}(t)\right]=\varepsilon(1-\varepsilon)
$$

\subsection{A Noise Model for Width Representation}

For width representation, only the leftmost and the rightmost pixels are needed. If there exist spurious pixels according to Eq. (4) in the background region, the leftmost pixel and rightmost pixel will be changed. According to the definition of width representation, the width vector will also be changed. If there exist salt-noise pixels in the background region with a lower $x$-coordinate value than that of the leftmost pixel, the width vector will be increased. Even when there is no saltnoise pixel in the background region, a width vector can still be decreased due to the consecutive pepper-noise pixels in a silhouette image. Let $\widehat{x_{h}^{R}}(t)$ and $\widehat{x_{h}^{L}}(t)$ denote the $x$-coordinate values of the rightmost non-zero pixel and the leftmost nonzero pixel generated by influence of noises in the background and foreground, respectively and $\lambda_{h}^{R}(t)$ and $\lambda_{h}^{L}(t)$ denote the left-side noise and the right-side noise, Then, the $h$ th element of the width vector $\widehat{w}(t)$ with noise at time $t$ is denoted as

$$
\begin{aligned}
\widehat{w_{h}}(t) & =\widehat{x_{h}^{R}}(t)-\widehat{x_{h}^{L}}(t) \\
& =\left(x_{h}^{R}(t)+\lambda_{h}^{R}(t)\right)-\left(x_{h}^{L}(t)+\lambda_{h}^{L}(t)\right) \\
& =\left(x_{h}^{R}(t)-x_{h}^{L}(t)\right)+\lambda_{h}(t) \\
& =w_{h}(t)+\lambda_{h}(t) .
\end{aligned}
$$

Here, $\lambda_{h}(t)=\lambda_{h}^{R}(t)-\lambda_{h}^{L}(t)$ is the total noise for a given row $h$.

The expectation of noise in width representation is evaluated by observing the sequence of pixels for a given row $h$. The addition of noise $\eta_{x y}(t)$ to an original silhouette image $I_{x y}(t)$ is considered and its distribution satisfies Eq. (4). The random variable $\widehat{x_{h}^{L}}(t)$ that interests us is $x$-coordinate value of the first non-zero pixel. For simplicity, $\widehat{x_{h}^{L}}(t)$ assume the values $1,2, \ldots$, $W / 2$ and we want to determine the corresponding probabilities.

First, we consider the probability that the pixel $k_{L}$ within the interval $\left[1, x_{h}^{L}(t)\right]$ is the leftmost pixel $\widehat{x_{h}^{L}}(t)$ due to noise for a given row $h$. This means that the noisy leftmost pixel is out of the silhouette region. Since only the leftmost pixel is considered in width representation, all other pixels within the interval $\left(k_{L}, x_{h}^{L}(t)\right]$ can be ignored. The first $k_{L}-1$ pixels should not be a noise pixel so that that $k_{L}$ th pixel is the leftmost pixel for a given row $h$. Hence, the probability that the noisy leftmost pixel is the $k_{L}$ th pixel is given by:

$$
P\left(\widehat{x_{h}^{L}}(t)=k_{L}\right)=(1-\varepsilon)^{k_{L}-1} \varepsilon, \quad 1 \leq k_{L} \leq x_{h}^{L}(t)
$$

and the left-side noise in width representation is given by:

$$
\lambda_{h}^{L}(t)=k_{L}-x_{h}^{L}(t)
$$

Next, we consider the probability that the pixel $k_{L}$ within the interval $\left(x_{h}^{L}(t), \frac{\mathrm{W}}{2}\right]$ is the leftmost pixel $\widehat{x_{h}^{L}}(t)$ due to noise for a given row $h$. This means that the noisy leftmost pixel is within the inner region of the silhouette. There is no salt noise pixel within the background region and consecutive pepper-noise pixels in inner region of silhouette from borderline between background and silhouette region. Hence, it has the probability

$P\left(\widehat{x_{h}^{L}}(t)=k_{L}\right)=(1-\varepsilon)^{x_{h}^{L}(t)-1} \varepsilon^{k_{L}-x_{h}^{L}(t)+1}, x_{h}^{L}(t)<k_{L} \leq \frac{\mathrm{W}}{2}$

and the left-side noise in width representation is given by: 


$$
\lambda_{h}^{L}(t)=k_{L}-x_{h}^{L}(t)+1 .
$$

The noise in terms of the leftmost pixel can thus be summarized as:

$$
\begin{aligned}
& P\left(\widehat{x_{h}^{L}}(t)=k_{L}\right) \\
& = \begin{cases}(1-\varepsilon)^{k_{L}-1} \varepsilon, & 1 \leq k_{L} \leq x_{h}^{L}(t), \\
(1-\varepsilon)^{x_{h}^{L}(t)-1} \varepsilon^{k_{L}-x_{h}^{L}(t)+1}, & x_{h}^{L}(t)<k_{L} \leq \frac{\mathrm{W}}{2},\end{cases} \\
& \lambda_{h}^{L}(t)= \begin{cases}k_{L}-x_{h}^{L}(t), & 1 \leq k_{L} \leq x_{h}^{L}(t), \\
k_{L}-x_{h}^{L}(t)+1, & x_{h}^{L}(t)<k_{L} \leq \frac{\mathrm{W}}{2} .\end{cases}
\end{aligned}
$$

Similarly, the noise in terms of the rightmost pixel can be formulated as:

$$
\begin{aligned}
& P\left(\widehat{x_{h}^{R}}(t)=k_{R}\right) \\
& = \begin{cases}(1-\varepsilon)^{\mathrm{W}-x_{h}^{R}(t)} \varepsilon^{x_{h}^{R}(t)-k_{R}+1}, & \frac{\mathrm{W}}{2}<k_{R}<x_{h}^{R}(t), \\
(1-\varepsilon)^{\mathrm{W}-k_{R}} \varepsilon, & x_{h}^{R}(t) \leq k_{R} \leq \mathrm{W},\end{cases} \\
& \lambda_{h}^{R}(t)= \begin{cases}k_{R}-x_{h}^{R}(t)-1, & \frac{\mathrm{W}}{2}<k_{R}<x_{h}^{R}(t), \\
k_{R}-x_{h}^{R}(t), & x_{h}^{R}(t) \leq k_{R} \leq \mathrm{W} .\end{cases}
\end{aligned}
$$

Consequently, the noise in width vector is defined as:

$$
\lambda_{h}(t)=\lambda_{h}^{R}(t)-\lambda_{h}^{L}(t)
$$

and

$$
\begin{aligned}
& E\left[\lambda_{h}(t)\right]= \sum_{k_{R}=\mathrm{W} / 2+1}^{\mathrm{W}} P\left(\widehat{x_{h}^{R}}(t)=k_{R}\right) \lambda_{h}^{R}(t) \\
&-\sum_{k_{L}=1}^{\mathrm{W} / 2} P\left(\widehat{x_{h}^{L}}(t)=k_{L}\right) \lambda_{h}^{L}(t) \\
&= \sum_{k_{r}=\mathrm{W} / 2+1}^{x_{h}^{R}(t)-1}\left((1-\epsilon)^{\mathrm{W}-x_{h}^{R}(t) \epsilon^{x_{h}^{R}(t)-k_{R}+1}} \times\left(k_{R}-x_{h}^{R}(t)-1\right)\right) \\
&+\sum_{k_{R}=x_{h}^{R}(t)}^{\mathrm{W}}(1-\epsilon)^{\mathrm{W}-k_{R}} \epsilon\left(k_{R}-x_{h}^{R}(t)\right) \\
&-\sum_{k_{L}=1}^{x_{h}^{L}(t)}(1-\epsilon)^{k_{L}-1} \epsilon\left(k_{L}-x_{h}^{L}(t)\right) \\
&-\sum_{k_{L}=x_{h}^{L}(t)+1}^{\mathrm{W} / 2}\left((1-\epsilon)^{x_{h}^{L}(t)-1} \epsilon^{k_{L}-x_{h}^{L}(t)+1}\right. \\
&\left.\times\left(k_{L}-x_{h}^{L}(t)+1\right)\right) .
\end{aligned}
$$

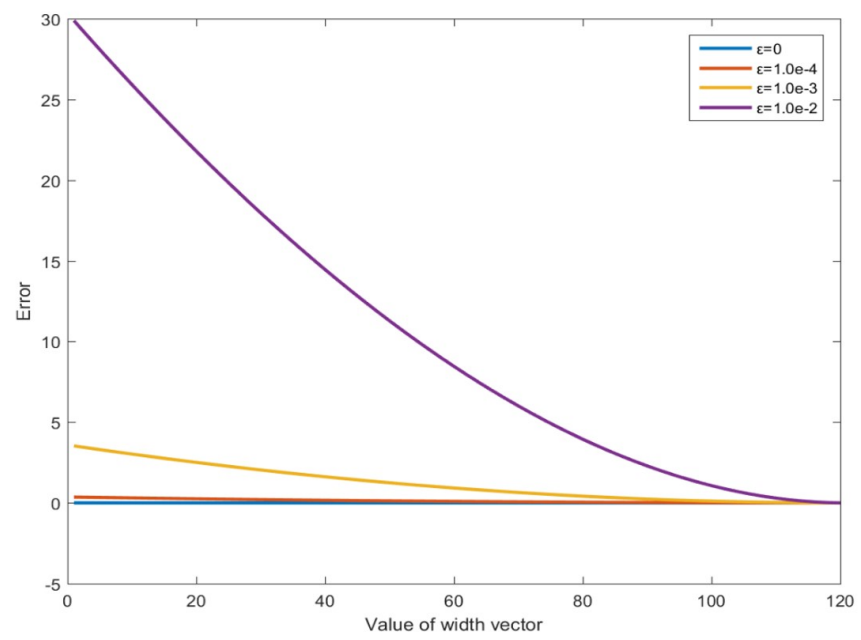

Figure 2. The expectation of noise in width representation in terms of the probability of noise within a silhouette image.

Here, the first and the fourth terms in Eq. (17) are small negative values and approach zero as the leftmost and rightmost pixels approach the $x$-coordinate center $\mathrm{W} / 2$.

Figure 2 shows the expectation of noise for width representations in terms of the probability within a silhouette image when the width of a normalized silhouette image is assumed to be 120. As shown in Figure 2, when the original value of the width vector extracted from individual silhouette without noise decreases, the expectation of noise increases exponentially. This means that the upper portion of body which can have small values of the width vector such as face, neck, and torso can be very sensitive to noise in a silhouette image. Figure 3 shows the example of the width vector contaminated with noise.

\section{Gait Recognition}

At the start, we parse a given binary gait sequence into gait cycles such that each cycle corresponds to one complete cycle from rest position to foot-forward-to-rest position. Figure 4 shows the norm of the width vector as a function of time for a given gait sequence. From its periodic characteristics, we can obtain $M$ keyframe indices, which are valleys or local minima corresponding to the rest position. We then extract the width vectors from each $M-1$ complete gait cycle and generate the mean of width vectors for classification.

The classification process is carried out via a simple classification method, namely, the nearest neighbor (NN) classifier. Euclidean distance is used for similarity measurement to resolve the matching problem. The mean of width vectors $\bar{w}_{(m)}$ 


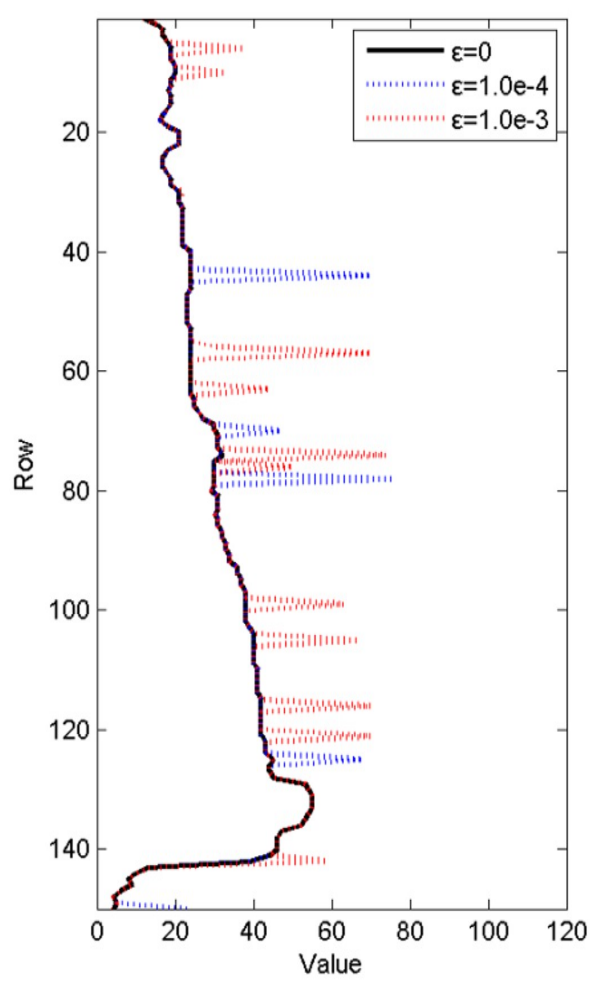

Figure 3. An example of noise contamination in width representation.

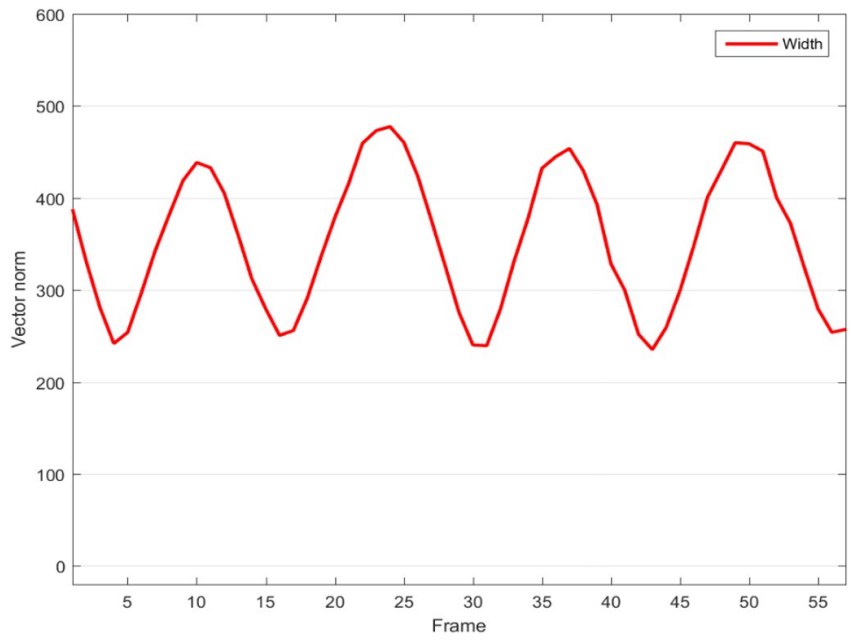

Figure 4. The norm of the width vector as a function of time.

obtained from the $m$ th complete gait cycle is classified as class $I D_{(m)}$ from minimizing the similarity measure given by:

$$
\begin{aligned}
& I D_{(m)}=\arg \min _{i} E\left(\bar{w}_{(m)}, \bar{w}_{c}^{n}\right) \\
& \quad \text { for } \forall n=1,2, \ldots, N_{c} \text { and } \forall c=1,2, \ldots, C,
\end{aligned}
$$

where $E(\bullet)$ is the Euclidean distance and $N_{c}$ is the number of means of width vectors in class $c$. At last, majority voting

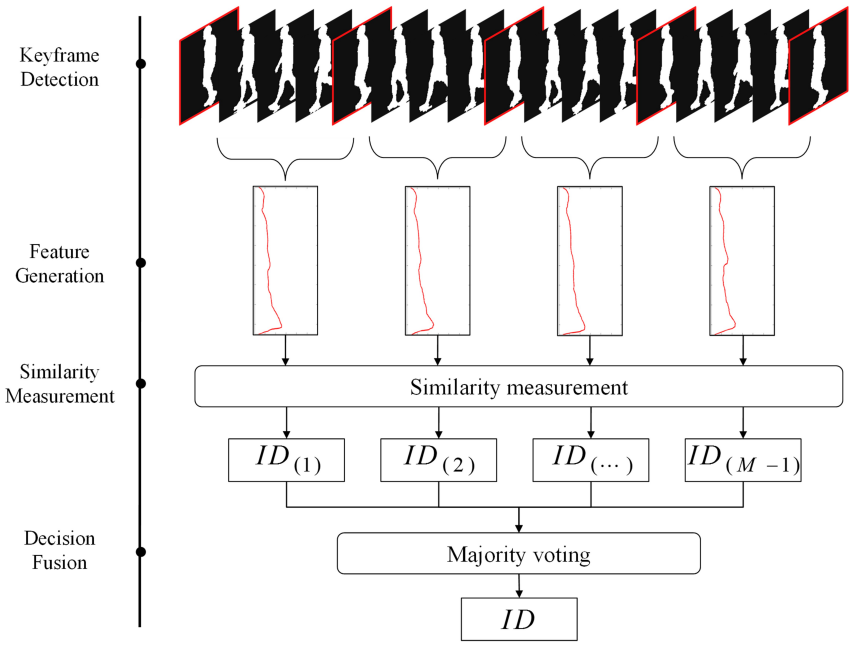

Figure 5. Procedure of the proposed gait recognition system based on decision fusion.

is used to obtain the final decision for a gait sequence. The proposed gait recognition system is shown in Figure 5.

\section{Experiments}

In this paper, two gait databases available in public domain are used to evaluate the recognition performance. The first database is the CASIA gait dataset $\mathrm{A}$ which is also known as the NLPR gait database [9] and the other database is the SOTON gait database [16]. The CASIA gait dataset A consists of 20 subjects and involves four sequences obtained from three different viewing cameras per subject, leading to a total of 80 sequences per view. In our experiment, we consider only canonical view. For a better statistical observation, we employ a larger SOTON database. This database contains over 100 subjects; thereby it is also referred to as the large-subject gait database. The SOTON gait database includes 113 different subjects with 4 sequences per subject where the images are captured at one canonical view.

In preprocessing, a binary foreground image involving human movements should extracted from an original visual image captured from a camera. Since both the CASIA gait database $\mathrm{A}$ and the SOTON database provide foreground images, any additional background subtraction technique is not required. Next, the silhouette normalization technique from [12] is used to reduce the effect of changes in silhouette size in walking. All silhouette images are normalized into $120 \times 150$ sizes.

The leave-one-out cross-validation test will be applied to the small-subject CASIA gait dataset A and the 4-fold crossvalidation test will be used for the large-subject SOTON gait 
Table 1. Correct classification rates (CCR) and false acceptance rates (FAR)

\begin{tabular}{ccc}
\hline Database & CCR & FAR \\
\hline CASIA A & 0.7125 & 0.0151 \\
SOTON & 0.8053 & 0.0017 \\
\hline
\end{tabular}

database to evaluate the recognition performance. Table 1 lists the correct classification rate (CCR) and the false acceptance rate (FAR) for the CASIA gait dataset A and the SOTON gait database when width representation has no noise, i.e., $\epsilon=0$.

Next, in order to illustrate the impact of noise to the recognition performance, the experiments are repeated while the probability of noise in Eq. (6) is increased from 0 to 0.02 . Figures 6 and 7 shows the CCRs and FARs with respect to the noise level added to the silhouette image for the CASIA gait dataset A and the SOTON gait database, respectively. As we expected, the performances of gait recognition using width representation start to degrade and are much affected by even a low level noise. Thus, it can be concluded that width representation is sensitive against the noise in silhouette image. Tables 2 and 3 summarize respectively the CCRs and FARs on the two gait database with respect to the noise level added to the silhouette image.

\section{Conclusion}

In this paper, gait representation based on the width of silhouette image is analyzed in terms of robustness against the noise in silhouette image. The expectation of the width vector for noises in silhouette image is mathematically derived based on a probabilistic noise model and experimentally verified through simulation. Besides, we presents a gait recognition system to divide a gait sequence into complete gait cycles using the periodic characteristics of width representation. Both the smallsubject CASIA gait dataset A and the large-subject SOTON gait database are used to evaluate the recognition performance with respect to the noise level added to the silhouette image. From the results, width representation has been much affected by even a low level noise. For future works, we analyze sensitivity of projection gait representation and improve the recognition performance.

\section{Conflict of Interest}

No potential conflict of interest relevant to this article was reported.

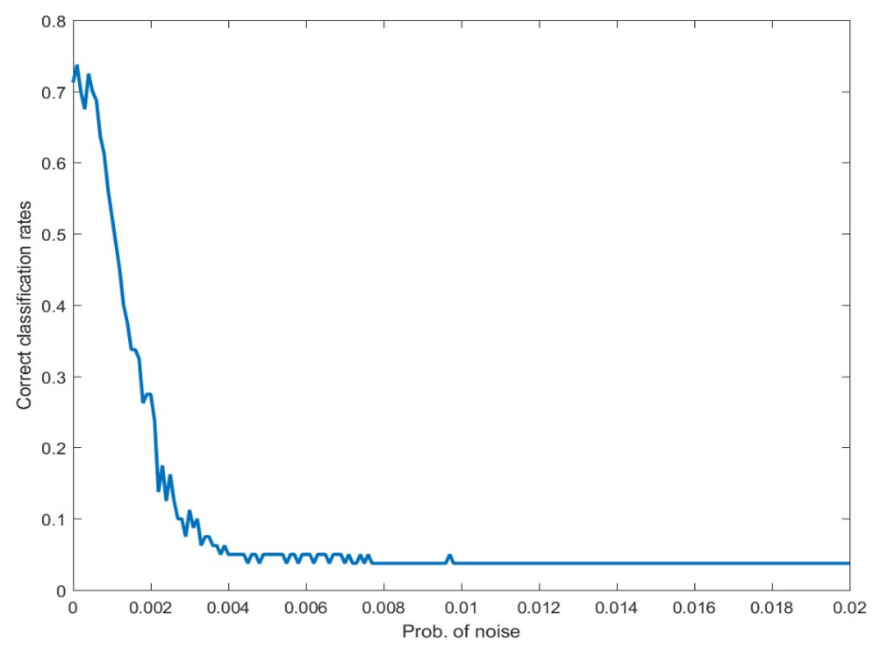

(a)

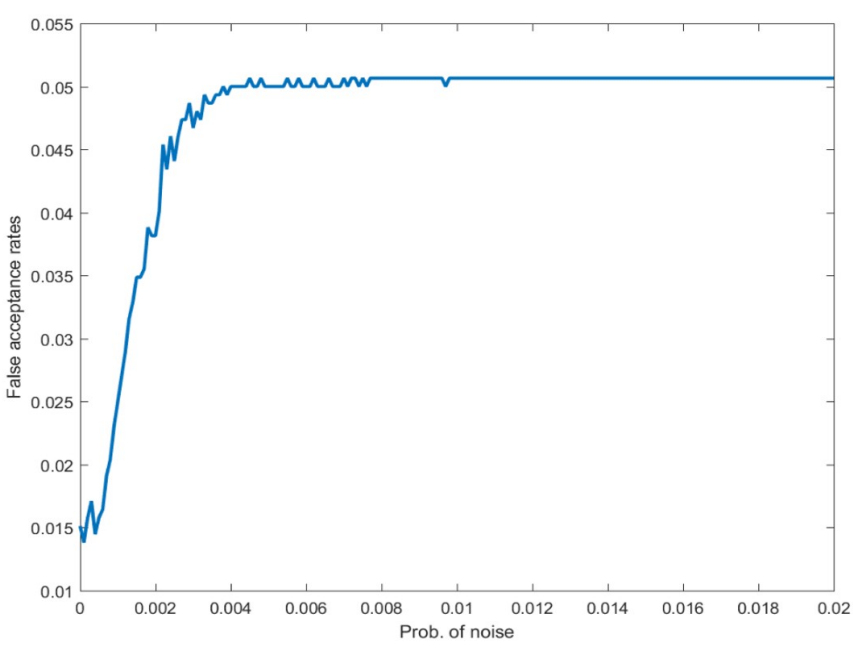

(b)

Figure 6. (a) Correct classification rates and (b) false acceptance rates versus the probability of noise for the CASIA gait dataset A.

\section{Acknowledgements}

This work was supported by the National Research Foundation of Korea (NRF) through the Biometrics Engineering Research Center (BERC) at Yonsei University in 2010 (Grant No. R112002-105-09002-0).

\section{References}

[1] M. H. Cheng, M. F. Ho, and C. L. Huang, "Gait analysis for human identification through manifold learning and HMM," Pattern Recognition, vol. 41, no. 8, pp. 2541-2553, 2008. http://dx.doi.org/10.1016/j.patcog.2007.11.021 
Table 2. Summary of correct classification rates

\begin{tabular}{cccccccccc}
\hline \multirow{2}{*}{ Database } & \multicolumn{8}{c}{ Probability of noise $(\varepsilon)$} \\
\cline { 2 - 10 } & 0.0000 & 0.0001 & 0.0002 & 0.0003 & 0.0004 & 0.0005 & 0.0050 & 0.0100 & 0.0200 \\
\hline CASIA A & 0.7125 & 0.7375 & 0.7000 & 0.6750 & 0.7250 & 0.7000 & 0.0500 & 0.0375 & 0.0375 \\
SOTON & 0.8053 & 0.7942 & 0.7721 & 0.7588 & 0.7146 & 0.6593 & 0.0111 & 0.0088 & 0.0088 \\
\hline
\end{tabular}

Table 3. Summary of false acceptance rates

\begin{tabular}{cccccccccc}
\hline \multirow{2}{*}{ Database } & \multicolumn{10}{c}{ Probability of noise $(\varepsilon)$} \\
\cline { 2 - 10 } & 0.0000 & 0.0001 & 0.0002 & 0.0003 & 0.0004 & 0.0005 & 0.0050 & 0.0100 & 0.0200 \\
\hline CASIA A & 0.0151 & 0.0138 & 0.0158 & 0.0171 & 0.0145 & 0.0158 & 0.0500 & 0.0507 & 0.0507 \\
SOTON & 0.0017 & 0.0018 & 0.0020 & 0.0022 & 0.0025 & 0.0030 & 0.0088 & 0.0088 & 0.0088 \\
\hline
\end{tabular}

[2] N. V. Boulgouris, D. Hatzinakos, and K. N. Plataniotis, "Gait recognition: a challenging signal processing technology for biometric identification," IEEE Signal Processing Magazine, vol. 22, no. 6, pp. 78-90, 2005. http://dx.doi.org/10.1109/MSP.2005.1550191

[3] A. Y. Johnson and A. F. Bobick, "A multi-view method for gait recognition using static body parameters," in Proceedings of 3rd International Conference on Audio- and Video-Based Biometric Person Authentication, Halmstad, Sweden, 2001, pp. 301-311. http://dx.doi.org/10.1007/3540-45344-X_44

[4] L. Lee and W. E. L. Grimson, "Gait analysis for recognition and classification," in Proceedings of 5th IEEE International Conference on Automatic Face and Gesture Recognition, Washington, DC, 2002, pp. 148-155. http://dx.doi.org/10.1109/AFGR.2002.1004148

[5] D. K. Wagg and M. S. Nixon, "On automated modelbased extraction and analysis of gait," in Proceedings of 6th IEEE International Conference on Automatic Face and Gesture Recognition, Seoul, Korea, 2004, pp. 11-16. http://dx.doi.org/10.1109/AFGR.2004.1301502

[6] J. Man and B. Bhanu, "Individual recognition using gait energy image," IEEE Transactions on Pattern Analysis and Machine Intelligence, vol. 28, no. 2, pp. 316-322, 2006. http://dx.doi.org/10.1109/TPAMI.2006.38

[7] X. Yang, Y. Zhou, T. Zhang, G. Shu, and J. Yang, "Gait recognition based on dynamic region analysis," Signal Processing, vol. 88, no. 9, pp. 2350-2356, 2008. http: //dx.doi.org/10.1016/j.sigpro.2008.03.006
[8] X. Li, S. J. Maybank, S. Yan, D. Tao, and D. Xu, "Gait components and their application to gender recognition," IEEE Transactions on Systems, Man, and Cybernetics, Part C (Applications and Reviews), vol. 38, no. 2, pp. 145-155, 2008. http://dx.doi.org/10.1109/TSMCC.2007. 913886

[9] L. Wang, T. Tan, H. Ning, and W. Hu, "Silhouette analysis-based gait recognition for human identification," IEEE Transactions on Pattern Analysis and Machine Intelligence, vol. 25, no. 12, pp. 1505-1518, 2003. http: //dx.doi.org/10.1109/TPAMI.2003.1251144

[10] A. Kale, N. Cuntoor, B. Yegnanarayana, A. N. Rajagopalan, and R. Chellappa, "Gait analysis for human identification," in Proceedings of 4th International Conference on Audio- and Video-Based Biometric Person Authentication, Guildford, UK, 2003, pp. 706-714. http: //dx.doi.org/10.1007/3-540-44887-X_82

[11] A. Kale, A. Sundaresan, A. N. Rajagopalan, N. P. Cuntoor, A. K. Roy-Chowdhury, V. Kruger, and R. Chellappa, "Identification of humans using gait," IEEE Transactions on Image Processing, vol. 13, no. 9, pp. 1163-1173, 2004. http://dx.doi.org/10.1109/TIP.2004.832865

[12] S. Hong, H. Lee, K. A. Toh, and E. Kim, "Gait recognition using multi-bipolarized contour vector," International Journal of Control, Automation, and Systems, vol. 7, no. 5, pp. 799-808, 2009. http://dx.doi.org/10.1007/s12555009-0512-1

[13] Y. X. Liu, R. Collins, and Y. H. Tsin, "Gait sequence analysis using frieze patterns," in Proceedings of 7th Euro- 


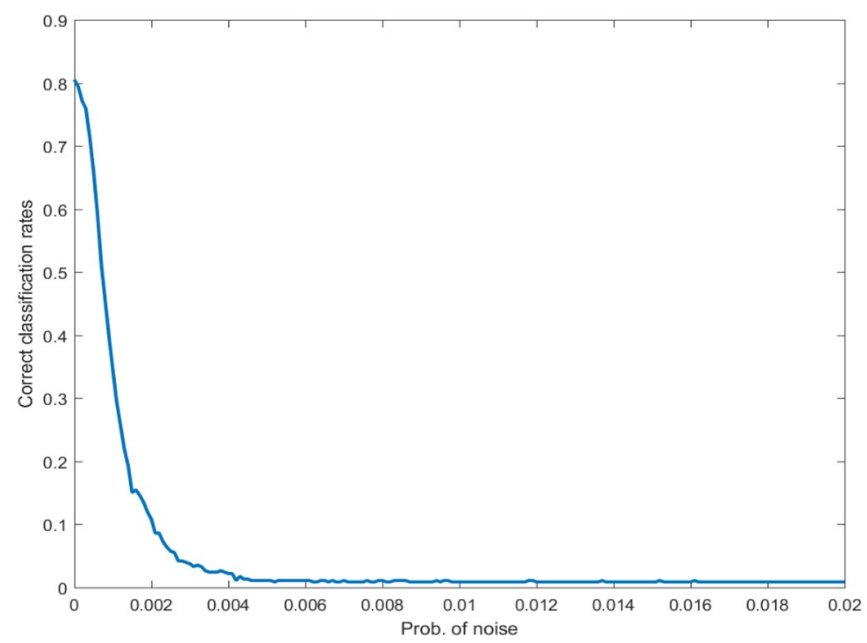

(a)

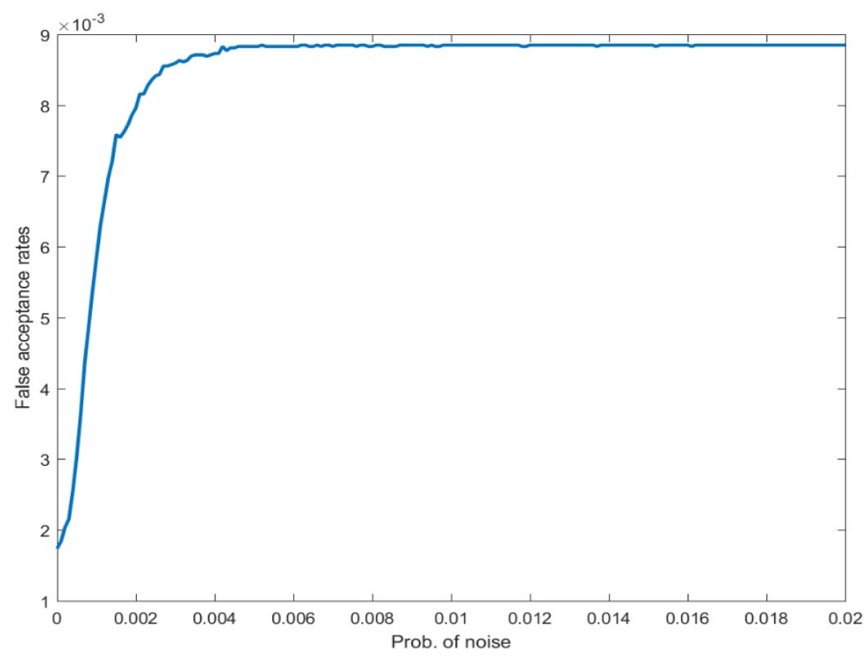

(b)

Figure 7. (a) Correct classification rates and (b) false acceptance rates versus the probability of noise for the SOTON gait database.

pean Conference on Computer Vision, Copenhagen, Denmark, 2002, pp. 657-671. http://dx.doi.org/10.1007/3-54047967-8_44

[14] S. Hong, H. Lee, I. F. Nizami, and E. Kim, "A new gait representation for human identification: mass vector," in Proceedings of 2007 2nd IEEE Conference on Industrial Electronics and Applications, Harbin, China, 2007, pp. 669-673. http://dx.doi.org/10.1109/ICIEA.2007.4318491

[15] T. H. W. Lam, K. H. Cheung, and J. N. K. Liu, "Gait flow image: a silhouette-based gait representation for human identification," Pattern Recognition, vol. 44, no. 4, pp. 973987, 2011. http://dx.doi.org/10.1016/j.patcog.2010.10.011

[16] J. D. Shutler, M. G. Grant, M. S. Nixon, and J. N. Carter, "On a large sequence-based human gait database," in Proceedings of the 4th International Conference on Recent Advances in Soft Computing, Nottingham, UK, 2002, pp. 66-71. http://dx.doi.org/10.1007/978-3-540-45240-9_46

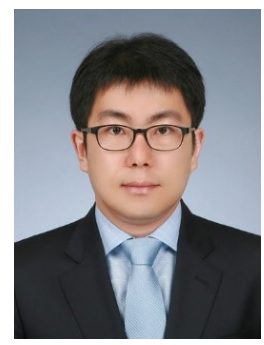

Sungjun Hong is a post-doctoral researcher in electrical and electronic engineering at Yonsei University, Seoul, Korea. He received the B.S. degree in electrical and electronic engineering and computer science (double major), and the Ph.D. degree in electrical and electronic engineering from Yonsei University in 2005 and 2012, respectively. Upon his graduation, he worked in the connected car industry in LG Electronics as a senior researcher from 2012 to 2013 . He worked for three years as a lead software engineer in SMARTSTUDY from 2013 to 2016 prior to his current appointment. He received the Institution of Engineering and Technology (IET) computer vision premium (best paper) award from the IET, UK in 2015. His current research interests include pattern recognition, machine learning, computer vision, and their application to autonomous vehicle, visual surveillance and biometrics.

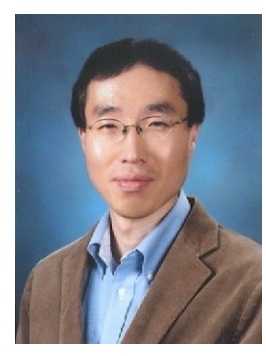

Euntai Kim received his B.S., M.S., and Ph.D. degrees in Electronic Engineering from Yonsei University, Seoul, Korea, in 1992, 1994, and 1999, respectively. He was a fulltime lecturer with the Department of Control and Instrumentation Engineering, Hankyong National University, Anseong, Korea from 1999 to 2002. Since 2002, he has been with the faculty of the School of Electrical and Electronic Engineering, Yonsei University, where he is currently a Professor. He was a visiting researcher at Berkeley Initiative in Soft Computing, UC at Berkeley, Berkeley, CA, USA. His current research interests include computational intelligence and statistical machine learning and their application to intelligent robot, vehicle, and machine vision. 